\title{
State estimation and fault detection of uncertain systems based on an interval approach
}

\author{
B. Marx, D. Maquin and J. Ragot
}

\begin{abstract}
This paper deals with state estimation and fault detection in the presence of unknown but bounded state perturbations and measurement noise. In this context, most available results are for linear models. Based on interval analysis, a state estimator for nonlinear dynamical systems is presented. Given the perturbation and noise bounds, the proposed method evaluates a set estimate guaranteed to contain all values of the state that are consistent with the available observations. The estimator is then used to regime shift detection. A numerical example is given.
\end{abstract}

\section{INTRODUCTION}

The aim of process diagnosis is to detect and identify faults affecting the process. The main idea of model-based fault diagnosis is to compare the behaviors of the process and its model when both are fed with the same inputs. As the process state is generally unknown, this comparison is generally achieved between the process output and the model output, this last being reconstructed by a state observer. State estimation using the exact knowledge of the input and output signals is well solved for processes with constant and known parameters (e.g. using a Luenberger or a finite memory observer). However, real processes are often affected by disturbances and noises which cause the generation of false alarms during the diagnosis. Therefore, state observer designs were extended to deal with disturbance and measurement noise.

However the situation becomes more critical when the considered system is subjected to unknown disturbances or unknown inputs. When the systems are subjected to perturbations with known statistic characteristics, Kalman's filter may be used to reconstruct the system state. In fact the observer design techniques for processes affected by uncertainties may be roughly divided into three groups.

The first group relies on robust estimation. The estimator is made robust to both exogenous signals (e.g. unknown inputs) and model uncertainties. In this framework, state estimation deals with the minimization of an induced norm (e.g. $\mathrm{H}_{2}$ or $H_{\infty}$ ) from disturbances to estimation errors.

In the second group, the state estimation is performed on a reduced system corresponding to the unknown input (UI) free subsystem (which exists under some restrictive conditions). For that, the state equation is splitted into two parts, one being sensitive to the UI, the other being decoupled from this input. It is then possible, under specific

\footnotetext{
The authors are with Centre de Recherche en Automatique de Nancy, UMR 7039, Nancy-Université CNRS, 2, Avenue de la Forêt de Haye,F 54516 Vandœuvre les Nancy \{benoit.marx, didier.maquin, jose.ragot densem.inpl-nancy.fr
}

conditions, to eliminate the UI influence on the state and the measurement equations by using an appropriate projection matrix, [5]. Another proposed observer design uses the sliding mode approach. Sliding observer is a high performance state estimator well adapted for nonlinear uncertain systems [14]. The sliding function of this observer is based on the estimation error of the available output of the system. Indeed, it uses a classical Luenberger observer with a nonlinear term depending on the estimation error.

The last group of methods relies on the description of the uncertainties by known compact sets. In the field of diagnosis, robust model-based fault detection of dynamic systems using interval observers has been already addressed. One of the main techniques consists in checking whether the measurements of the output belong to the interval of all possible estimated outputs obtained considering uncertainty on model parameters [15], [10], [13]. Original applications in the field of flow rate sensor diagnosis and data validation are presented in [11] and [1]. Although interval approaches need very little a priori information (only the uncertain parameter bounds), only few published works are dedicated to process diagnosis, like [9] or [12].

The paper is organized as follows: after a brief overview, in section 2, the ideal observer structure is defined. In section 3 , fault diagnosis techniques for nonlinear systems are derived from the estimation method. The last section is devoted to a numerical example dealing with the determination of the active mode of a system under supervision.

Notations. In this study, only real intervals are considered. As a definition, a real interval, denoted $[x]$, is a closed and connected subset of $\mathbb{R}$, defined by:

$$
[x]=\left[x^{-} x^{+}\right]=\left\{x \in \mathbb{R} / x^{-} \leq x \leq x^{+}\right\}
$$

This definition can be extended to the $v$-dimension space: an interval vector $[x]$ of $\mathbb{R}^{v}$ is an $v$-dimensional rectangle or "box" of $\mathbb{R}^{v}$ and is the Cartesian product of intervals. The set of all boxes of $\mathbb{R}^{v}$ is denoted $\mathbb{I} \mathbb{R}^{v}$.

\section{A. Problem formulation}

The behavior of the system is described by a discrete-time uncertain nonlinear model. The uncertainties are induced by a vector of interval parameters $\theta(k)$, and by the interval disturbances $v(k)$ and $w(k)$ affecting the state and measurement equations respectively:

$$
S \quad\left\{\begin{aligned}
x(k+1) & =f(x(k), u(k), \theta(k), v(k)) \\
y(k) & =h(x(k), \theta(k))+w(k)
\end{aligned}\right.
$$


where $f$ and $h$ are known nonlinear functions and $x$ is the state, $y$ is the output, $u \in \mathbb{R}^{r}$ is the input, $v \in \mathbb{I}^{n_{v}}$ and $w \in$ $\mathbb{I} \mathbb{R}^{n_{w}}$ are input and output disturbances. Since the parameter vector $\theta(k)$ and the disturbances $v(k)$ and $w(k)$ are interval and neglecting the coupling between the state variables and the outputs, the state and output vectors will be bounded by some boxes, $x \in \mathbb{R}^{n}$ and $y \in \mathbb{I}^{p}$. The interval parameters allow to take into account the evolution of the system i.e. changing operation point. The initial state $x(0)$ is assumed to belong to some a priori known compact set $\mathcal{D}_{x, 0} \in \mathbb{R}^{n}$. The sequences $\{\theta(k)\},\{v(k)\}$ and $\{w(k)\}$ are unknown, but are assumed to belong to known compact sets:

$$
X^{-}(k) \leq X(k) \leq X^{+}(k), \quad X \in\{\theta, v, w\}
$$

where inequalities are element wise inequality and where the indicated bounds are known at each time $k$. It should be noticed that, excepted the input $u(k)$, all the signals are intervals, including the outputs of the model. Nevertheless the measured output, $y_{m}(k) \in \mathbb{R}^{p}$, is not an interval. It should be understood in the sense that the measured output belongs to the interval $y(k)$ which stands for all the possible output values consistent with the model.

A guaranteed state estimator, also named set-valued observer (SVO) constructs sets of admissible states which are consistent with the a priori bounds $v^{-}, v^{+}, w^{-}, w^{+}$.

The main idea for that construction consists in determining the set of the possible state values which are consistent with the known bounds of the uncertainties, the model equation and the current measurements (i.e. the admissible domain). The book [6] presents the basic statements of such methods. More recent works may be found in [7].

\section{IDEAL OBSERVER}

The first objective is to estimate the state of the system (1). The state estimation can be obtained, on the one hand, by inversing the output equation of (1) or, on the other hand, by recursively integrating the state equation of (1). The former is based on the measurements $y_{m}(k)$ and the bounds of the output disturbances $w^{-}(k)$ and $w^{+}(k)$. The latter is based on the knowledge of the previous estimation, the input $u(k-$ 1) and the bounds of the uncertainties and the disturbances $\theta^{-}(k-1), \theta^{+}(k-1), v^{-}(k-1), v^{+}(k-1)$. Obviously, the sought estimated interval of the state values, denoted $\mathcal{D}_{x, k}$, must be consistent with both the measurements and the model, which leads to compute the intersection of the intervals obtained by the two methods.

To begin with, the initial state $x(0)$ of system (1) is assumed to belong to the compact set $\mathcal{D}_{x, 0} \in \mathbb{R}^{n}$. Then, for a given $k, \mathcal{D}_{x, k}$ represents the set of all the state values, $x(k)$, consistent with the available informations $I_{k}$ :

$$
\begin{aligned}
I_{k}= & \left\{\mathcal{D}_{x, 0},\left\{u(i), y_{m}(i), \theta^{-}(i), \theta^{+}(i), v^{-}(i), v^{+}(i),\right.\right. \\
& \left.\left.w^{-}(i), w^{+}(i)\right\}_{i=1}^{k}\right\}
\end{aligned}
$$

The estimation process is detailed in the following steps:

- Observation step. The observation allows to deduce the possible values of the state $x(k)$ consistent with the measurement $y_{m}(k)$. First, the sets of the output values which are consistent with the measurements $y_{m}(k)$ and the uncertainty bounds are defined by:

$$
\mathcal{D}_{y, k}=\left\{y \in \mathbb{I}^{p} / w^{-}(k) \leq y-y_{m}(k) \leq w^{+}(k)\right\}
$$

The set of possible state values $x(k)$ is deduced from:

$$
\begin{aligned}
\mathcal{D}_{x, k}^{y}= & \left\{x \in \mathbb{I}^{n} / h(x, \theta(k)) \in \mathcal{D}_{y, k},\right. \\
& \left.\theta^{-}(k) \leq \theta(k) \leq \theta^{+}(k)\right\}
\end{aligned}
$$

In words, $\mathcal{D}_{x, k}^{y}$ contains all state values $x(k)$ which could have led to the observation $y_{m}(k)$. In most situations, numerical algorithms are used to approximate the true domain with guarantee to enclose it.

- Prediction step. This step involves the evaluation of the state equation to propagate the current set of estimated states and parameters. The obtained domain, denoted $\mathcal{D}_{x, k}^{+}$, is the set of possible $x(k+1)$, predicted by the state equation, computed with measurement and estimation up to time $k$ :

$$
\begin{aligned}
\mathcal{D}_{x, k}^{+}= & \left\{f(x, u(k), \theta, v) / x \in \mathcal{D}_{x, k}, v^{-}(k) \leq v \leq v^{+}(k),\right. \\
& \left.\theta^{-}(k) \leq \theta \leq \theta^{+}(k)\right\}
\end{aligned}
$$

- Correction step. Finally, the admissible domain $\mathcal{D}_{x, k+1}$ compatible with the information $I_{k+1}$ is obtained by the intersection of the two domains obtained using the model equation and the measurement equation, as follows:

$$
\mathcal{D}_{x, k+1}=\mathcal{D}_{x, k}^{+} \cap \mathcal{D}_{x, k+1}^{y}
$$

Remark 1. In some situation, depending on the measurement values, it is possible to obtain an empty intersection when using (4) because of contradictory knowledges. If necessary, this problem may be overcome by using an expansion operator allowing to enlarge the domain of the admissible output. However, when the objective of the state estimation is included in the general framework of diagnosis, this particular situation may be analyzed in order to detect and identify faults.

Remark 2. Repeated applications of the intersection procedure (4) generally leads to a complex shape (and consequently a complex description) of the set $\mathcal{D}_{x, k}$ and may be serious drawbacks for real-time application. To overcome this problem, it is possible to reduce the complexity of the procedure by evaluating an approximated (but guaranteed) set $\hat{\mathcal{D}}_{x, k}$ of $\mathcal{D}_{x, k}$, as suggested in several papers (e.g. [3]). Consequently, the SVO algorithm is structured as follow:

Algorithm 1: state estimation

- Step 0. Initialize $\mathcal{D}_{x, 0}^{+}$with $\mathcal{D}_{0}$. Let $k=1$

- Step 1. Collect the data $u(k)$ and $y_{m}(k)$

- Step 2. Compute the output domain $\mathcal{D}_{y, k}$ :

$$
\mathcal{D}_{y, k}=\left\{y / w^{-}(k) \leq y-y_{m}(k) \leq w^{+}(k)\right\}
$$

- Step 3. Compute the state domain $\mathcal{D}_{x, k}^{y}$ :

$\mathcal{D}_{x, k}^{y}=\left\{x \in \mathbb{I}^{n} / h(x, \theta(k)) \in \mathcal{D}_{y, k}, \theta^{-}(k) \leq \theta(k) \leq \theta^{+}(k)\right\}$ 
- Step 4. Compute the admissible state domain $\mathcal{D}_{x, k}$ :

$$
\mathcal{D}_{x, k}=\mathcal{D}_{x, k-1}^{+} \cap \mathcal{D}_{x, k}^{y}
$$

- Step 5. Reduce the domain complexity:

$$
\hat{\mathcal{D}}_{x, k} \supseteq \mathcal{D}_{x, k}
$$

- Step 6. Predict the state set

$$
\begin{aligned}
\mathcal{D}_{x, k}^{+}= & \left\{f(x, u(k), \theta, v) / x \in \hat{\mathcal{D}}_{x, k}, v^{-}(k) \leq v \leq v^{+}(k),\right. \\
& \left.\theta^{-}(k) \leq \theta \leq \theta^{+}(k)\right\}
\end{aligned}
$$

- Step 7. Increase $k:=k+1$, go to Step 1

\section{APPLICATION TO DIAGNOSIS}

When applying the previous procedure, one assumes implicitly that both sources of information are coherent. For diagnosis purpose, it is precisely the problem of inconsistency of information which prevails. The impossibility of merging the two sources of information (the two domains $\mathcal{D}_{x, k-1}^{+}$and $\mathcal{D}_{x, k}^{y}$ ) reveals the incompatibility between the measurements and the model of the system. During this diagnosis analysis, one is particularly interested in estimating the system outputs to be compared with the measured outputs in order to generate the so-called residuals, whereas this analysis is not possible for the system state.

\section{A. Principle of fault detection}

The output domain $\mathcal{D}_{y, k}^{+}$predicted by the model of the system is deduced from the state domain $\mathcal{D}_{x, k}^{+}$which has been already defined (see algorithm 1):

$$
\begin{array}{r}
\mathcal{D}_{y, k}^{+}=\left\{y^{+} / y^{+}=h\left(x^{+}, \tilde{\theta}\right)+w, x^{+}=f(x, u(k), \theta, v),\right. \\
x \in \mathcal{D}_{x, k}, \theta^{-}(k+1) \leq \tilde{\theta} \leq \theta^{+}(k+1), \theta^{-}(k) \leq \theta \leq \theta^{+}(k), \\
\left.v^{-}(k) \leq v \leq v^{+}(k), w^{-}(k) \leq w \leq w^{+}(k)\right\}
\end{array}
$$

In the same way, the admissible output domain $\mathcal{D}_{y, k+1}$, evaluated from the measurements, is defined by:

$$
\mathcal{D}_{y, k+1}=\left\{y / w^{-}(k) \leq y-y_{m}(k+1) \leq w^{+}(k)\right\}
$$

Consequently, starting from these two domains, a fault indicator can be defined. Let us consider:

$$
r_{k+1}=\mathcal{D}_{y, k}^{+} \cap \mathcal{D}_{y, k+1}
$$

A fault is detected if $r_{k+1}=\emptyset$ (equality is element wise equatlity and thus a fault is detected if at least one component of $r$ is the empty set). One should note that, determining the frontiers of the two domains at every time may result in an important computational load. For this reason, the exact domain is often approximated by a domain of simpler form, for example presenting less vertice. Thus, modifying (7), a fault is detected if the following residual is empty:

$$
\hat{r}_{k+1}=\hat{\mathcal{D}}_{y, k}^{+} \cap \mathcal{D}_{y, k+1}
$$

where $\hat{\mathcal{D}}_{y, k}^{+}$is an overestimation of $\mathcal{D}_{y, k}^{+}$, i.e.:

$$
\mathcal{D}_{y, k}^{+} \subseteq \hat{\mathcal{D}}_{y, k}^{+}
$$

If $\hat{\mathcal{D}}_{y, k}^{+}$is easier to compute than $\mathcal{D}_{y, k}^{+}$, the residuals (8) are simpler to compute that (7); however, the domain $\hat{\mathcal{D}}_{y, k}^{+}$ results to detect less faults than the domain $\mathcal{D}_{y, k}^{+}$. In fact, if $\hat{\mathcal{D}}_{y, k}^{+} \cap \mathcal{D}_{y, k+1} \neq \emptyset$ and $\mathcal{D}_{y, k}^{+} \cap \mathcal{D}_{y, k+1}=\emptyset$, then a fault occurred but was not detected [2]. Thus the difficulty is to define a compromise between the complexity of the determination of the state domain or the output domain and the tolerable rate of no detection. The reader will notice that, compared to what was presented at the section II, within the framework of the state estimation, the reduction of complexity was not carried out on the same domain. According to the difficulty of implementation, the user can choose to do this reduction at any step of the proposed algorithms, keeping in mind that this latter always generates an approximation.

The preceding formalism makes it possible to detect inconsistencies of data. Nevertheless, this diagnosis remains a little vague, thus it is worth specifying how, in a more general way, to highlight the occurrence of a fault. A solution consists in computing the interval state estimate using only a part of the output measurements. Analogously to the design of banks of dedicated observers in [4], $p$ domains can be built, where each domain is computed with only one component of the measurement vector $y_{m}$.

\section{B. Principle for change detection of operating mode}

In the framework of supervised diagnosis, one admits that all the failures affecting a system have been listed and associated to a known model. Therefore, each normal operating mode or dysfunctioning mode is thus described by a model.

$$
M_{i}\left\{\begin{aligned}
x_{i}(k+1) & =f_{i}\left(x_{i}(k), u(k), \theta(k), v(k)\right) \\
y_{i}(k) & =h_{i}\left(x_{i}(k), \theta(k)\right)+w(k)
\end{aligned}\right.
$$

where $v$ and $w$ already denotes the uncertainties affecting the model and the measurement system.

In a more general way, the set of the models $M_{i}, \quad i=0 \ldots N$ represents all the operating modes including the healthy modes related to the absence of faults. Thus, the diagnosis consists, starting from available measurements, in determining which model, among a set of models, is compatible with the measurements and the bounds of the uncertainties. In our case, the selected principle is the invalidation of model. At any moment $k$, each model $M_{i}$ allows to predict the state $x$ in an interval form (defining a domain $\mathcal{D}_{x, k, i}^{+}$, where the subscript $i$ is related to the number of the model). If a prediction is incompatible with the state $\mathcal{D}_{x, k+1, i}^{y}$ deduced from the measurements $y$, then the corresponding model does not reflect the current situation and thus the system does not operate in the corresponding mode. The algorithm to be implemented, inspired of [9], is then the following:

Algorithm 2: state estimation (multiple modes)

- Step 0. Define the initial state domains $\mathcal{D}_{x, 0}^{+}$, and set $k=1$.

- Step 1. Collect the data $u(k)$ and $y_{m}(k)$ 
- Step 2. Compute the output domains $\mathcal{D}_{y, k, i}$, for $i=$ $0, \ldots, N$,

$$
\mathcal{D}_{y, k, i}=\left\{y / w_{i}^{-}(k) \leq y-y_{m}(k) \leq w_{i}^{+}(k)\right\}
$$

- Step 3. Compute the admissible state domains starting from the output domain, for $i=0, \ldots, N$ :

$$
\mathcal{D}_{x, k, i}^{y}=\left\{x \in \mathbb{I R}^{n} / h_{i}(x, \theta) \in \mathcal{D}_{y, k, i}, \theta^{-}(k) \leq \theta \leq \theta^{+}(k)\right\}
$$

- Step 4. Compute, at the moment $k$, the admissible state domains $\mathcal{D}_{x, k, i}$, for $i=0, \ldots, N$ :

$$
\mathcal{D}_{x, k, i}=\mathcal{D}_{x, k-1, i}^{+} \cap \mathcal{D}_{x, k, i}^{y}
$$

- Step 5. Analyse the domains $\mathcal{D}_{x, k-1, i}$. If $\mathcal{D}_{x, k-1, i_{0}}=\emptyset$, the $i_{0}$ is not an active mode

- Step 6. Reduce the domains complexity, for $i=0, \ldots, N$ :

$$
\mathcal{D}_{x, k, i}^{+} \subseteq \hat{\mathcal{D}}_{x, k, i}^{+}
$$

- Step 7. Characterize the admissible state domains using prediction based on the $i^{t h}$ model, for $i=0, \ldots, N$ :

$$
\begin{aligned}
\mathcal{D}_{x, k, i}^{+}= & \left\{f_{i}(x, u(k), \theta, v) / x \in \hat{\mathcal{D}}_{x, k, i},\right. \\
& \left.v^{-}(k) \leq v \leq v^{+}(k), \theta^{-}(k) \leq \theta \leq \theta^{+}(k)\right\}
\end{aligned}
$$

- Step 8. Increase $k=k+1$ and go to Step 1 .

The interpretation of the various domains $\mathcal{D}_{x, k, i}$ is done in the following way. Let us recall that $\mathcal{D}_{x, k, i}, i=0, \ldots, N$ is the set of all the admissible states consistent with the measurements and the uncertainty bounds, considering the $i^{t h}$ model. If $\mathcal{D}_{x, k, i_{0}}$ is empty, it means that the current evolution is not correctly described by the $i_{0}^{\text {th }}$ mode.

Obviously, if the domains $\mathcal{D}_{x, k, i_{1}}$ and $\mathcal{D}_{x, k, i_{2}}$ are not simultaneously empty, there is an ambiguity. Indeed, the two modes $i_{1}$ et $i_{2}$ are candidates to describe the corresponding situation. In this case, additional information is necessary to refine the diagnosis and to distinguish the modes $i_{1}$ and $i_{2}$. The concept of persistence can be a useful recourse. The method is to build and analyze the various domains at consecutive moments, the vacuity of the domains is then analyzed over a more significant duration.

\section{Residual generation and diagnostic}

\section{Algorithm 3: diagnosis (multiple modes)}

- Step 0. Define the initial state domains $\mathcal{D}_{x, 0}^{+}$, and set $k=1$.

- Step 1. Collect the data $u(k)$ and $y_{m}(k)$.

- Step 2. Characterize the admissible state domains using a prediction based on the $i^{t h}$ model, for $i=0, \ldots, N$ :

$$
\begin{aligned}
\mathcal{D}_{x, k, i}^{+}= & \left\{f_{i}(x, u(k), \theta, v) / x \in \hat{\mathcal{D}}_{x, k, i}, \theta^{-}(k) \leq \theta \leq \theta^{+}(k),\right. \\
& \left.v^{-}(k) \leq v \leq v^{+}(k)\right\}
\end{aligned}
$$

- Step 3. Characterize the admissible output domains $\mathcal{D}_{y, k, i}^{+}$

- Step 4. Compute the bounds of the output domains

$$
\left\{\begin{array}{l}
y_{i j}^{-}(k)=\inf y / y \in \mathcal{D}_{y, k, i}^{+} \\
y_{i j}^{+}(k)=\sup y / y \in \mathcal{D}_{y, k, i}^{+}
\end{array}\right.
$$

where the $j$ is the number of the component output.

- Step 5. Compute the interval residuals

$$
\left[r_{i j}(k)\right]=\left[y_{i j}^{-}(k)-y_{m j}(k), y_{i j}^{+}(k)-y_{m j}(k)\right]
$$

- Step 6. Test the residual by checking if:

$$
0 \in\left[r_{i j}(k)\right], i=0 \ldots N, j=1, \ldots, p
$$

- Step 7. Increase $k=k+1$ and go to Step 1 .

The active mode detection is computed, using the residual signals as follows. For a given $k$, the $i^{\text {th }}$ mode is said

- not active, if $0 \notin\left[r_{i j}(k)\right], \exists j \in\{1, \ldots, p\}$

- active, if $0 \in\left[r_{i j}(k)\right], \forall j \in\{1, \ldots, p\}$

\section{EXAMPLE: SEARCH FOR ACTIVE MODE}

Let us consider a system which can be in a normal operation mode $(i=0)$ or in two abnormal modes of dysfunction $(i=1,2)$. It is assumed that the three corresponding models and the measurements of the inputs and outputs are known. The arising problem is to determine the current operating mode of the system at every moment.

\section{A. System models}

To simplify the presentation of the numerical results, the three models are taken as linear relations between the input $u$ and the state $x \in \mathbb{R}^{2}$ and as nonlinear relations between the state $x$ and the output $y_{i}=\left(\begin{array}{ll}y_{i 1} & y_{i 2}\end{array}\right)^{T} \in \mathbb{R}^{2}$. At each time $k$, the system is running under a particular mode (characterized by a particular function $h_{i}$ ) according to an external or internal variable.

$$
M_{i}\left\{\begin{aligned}
x(k+1) & =A x(k)+B u(k)+F v(k) \\
y_{i}(k) & =h_{i}(x(k), \theta)+w(k),
\end{aligned}\right.
$$

with

$$
\begin{gathered}
A=\left(\begin{array}{cc}
0.6 & 0 \\
-0.2 & 0.5
\end{array}\right), \quad B=\left(\begin{array}{l}
1 \\
0
\end{array}\right), \quad F=\left(\begin{array}{cc}
0.05 & 0 \\
0 & 0.05
\end{array}\right) \\
h_{0}(x(k), \theta(k))=\left(\begin{array}{c}
\frac{x_{1}(k)+\theta_{1}(k)}{1+\theta_{2}(k) x_{1}(k)} \\
\frac{x_{1}(k)+x_{2}(k)+\theta_{2}(k)}{\theta_{1}(k)+x_{2}^{2}(k)}
\end{array}\right) \\
h_{1}(x(k), \theta(k))=\left(\begin{array}{c}
\frac{x_{1}(k)+0.5+\theta_{1}(k)}{1+\left(\theta_{2}(k)-0.5\right) x_{1}(k)} \\
\frac{x_{1}(k)+x_{2}(k)-0.5+\theta_{2}(k)}{0.5+\theta_{1}(k)+x_{2}^{2}(k)}
\end{array}\right) \\
h_{2}(x(k), \theta(k))=\left(\begin{array}{c}
\frac{x_{1}(k)+1.5 \theta_{1}(k)}{1+\left(0.5+3 \theta_{2}(k)\right) x_{1}(k)} \\
\frac{x_{1}(k)+x_{2}(k)+0.5+3 \theta_{2}(k)}{1.5 \theta_{1}(k)+x_{2}^{2}(k)}
\end{array}\right) \\
-1 \leq v_{i}(k) \leq 1, \quad-0.04 \leq w_{i}(k) \leq 0.04 \\
0.8 \leq \theta_{1}(k) \leq 1.2 \quad 1.3 \leq \theta_{2}(k) \leq 1.7
\end{gathered}
$$

Although the system has varying parameters, it is desired to detect, at each time $k$, in which mode the system lies.

\section{B. Improving, or not, the output estimation}

The system output $y(k)$ can be predicted from the input $x(k)$ using each of the three operating models:

$$
\begin{aligned}
\mathcal{D}_{y, k, i}= & \left\{y / y=f_{i}(x(k), \theta)+w, \theta^{-}(k) \leq \theta \leq \theta^{+}(k),\right. \\
& \left.w^{-}(k) \leq w \leq w^{+}(k)\right\}
\end{aligned}
$$



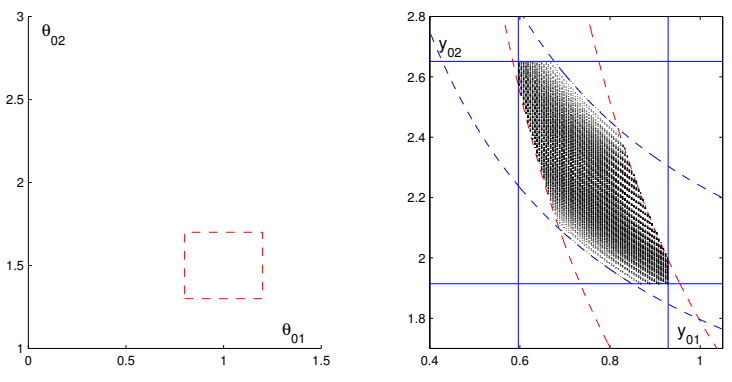

Fig. 1. Parameter and output domains ( $M_{0}$ model).

The figure 1 illustrates the $\mathcal{D}_{y, k, 0}$ domain construction, at a particular time $k_{0}$, for which the state is defined by $x\left(k_{0}\right)=$ $\left[\begin{array}{ll}2 & 1\end{array}\right]^{T}$. In this case, the parameter polytope, defined by a set of inequalities, is drawn in the plan of the components of $\theta$ on the left side of figure 1 . The two other polytopes relating to the parametric domains of the models $M_{1}$ and $M_{2}$ may be built in a similar way.

The outputs $y_{m 01}$ and $y_{m 02}(15)$ are given by:

$$
\left\{\begin{array}{l}
y_{m 01}\left(k_{0}\right)=\frac{1.5+\theta_{1}\left(k_{0}\right)}{1+2 \theta_{2}\left(k_{0}\right)}+w_{1}\left(k_{0}\right) \\
y_{m 02}\left(k_{0}\right)=\frac{3+\theta_{2}\left(k_{0}\right)}{1+\theta_{1}\left(k_{0}\right)}+w_{2}\left(k_{0}\right)
\end{array}\right.
$$

which has been represented on the right part of figure 2 . Taking into account the bounds of $\theta$, one obtains $y_{0 m 1}\left(k_{0}\right)=$ $\left[\begin{array}{ll}0.596 & 0.929\end{array}\right]$ and $y_{0 m 2}\left(k_{0}\right)=\left[\begin{array}{ll}1.915 & 2.651\end{array}\right]$. However, it should be noticed that $y_{0 \mathrm{~m} 1}$ and $y_{0 \mathrm{~m} 2}$ are coupled via the two standardized uncertainties $\eta_{1}$ and $\eta_{2}$. The couplings are highlighted by eliminating $\theta_{1}\left(k_{0}\right)$ or $\theta_{2}\left(k_{0}\right)$ in $(15)$, which can be rewritten more explicitly as:

$$
y_{0}\left(k_{0}\right)=\left(\begin{array}{c}
\frac{2+\theta_{1}\left(k_{0}\right)}{2\left(y_{02}\left(k_{0}\right)-w_{2}\left(k_{0}\right)\right)\left(1+\theta_{1}\left(k_{0}\right)\right)}+w_{1}\left(k_{0}\right) \\
\frac{3+\theta_{2}\left(k_{0}\right)}{\left(1+2 \theta_{2}\left(k_{0}\right)\right)\left(y_{01}\left(k_{0}\right)-w_{1}\left(k_{0}\right)\right)-1}+w_{2}\left(k_{0}\right)
\end{array}\right)
$$

The domain corresponding to this description is represented on the figure 1 in the plan of the outputs. The "complex" shape of this domain (grayed zone of the right part of the figure) results from (15) and (18). In the sequel, in order to simplify the fault detection procedure, the selected domain is the smallest orthotope $\square_{y, k, 0}$ containing the exact domain, defined by (15). In other words the coupling between the outputs is not taken into account. Same construction applies to the polytopes resulting from the two other models. Finally, one can state that: to the moment considered, the vector parameter $\theta$ belongs to one of the three polytopes of the figure 2 (left-hand side). For this particular example, one can note that the three zonotopes $\square_{y, k, i}, i=1,2,3$ (right-hand side of the figure) are almost totally disjoined.

\section{Generation of the active mode indicators}

The previous construction, taking into account available measurements is carried out on-line, after each acquisition of measurements. In order to avoid the representation of the domains obtained at every moment for every model, an interval representation is now adopted. The interval $\left[y_{i j}^{-}(k) y_{i j}^{+}(k)\right]$ denotes the bounds of $y_{i j}(k)$ the $j^{t h}$ component of the output
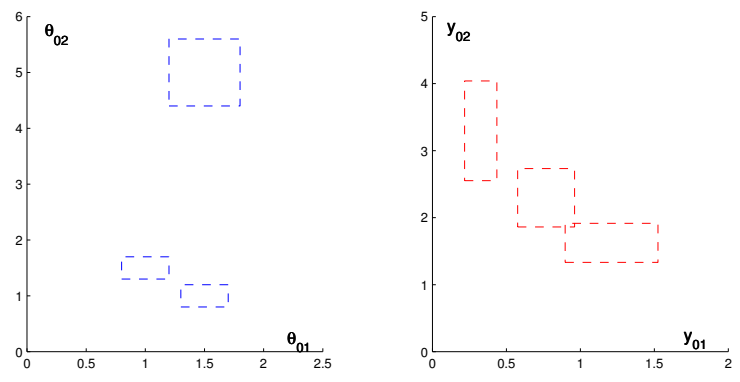

Fig. 2. Parameter and output domains ( $M_{i}$ models).
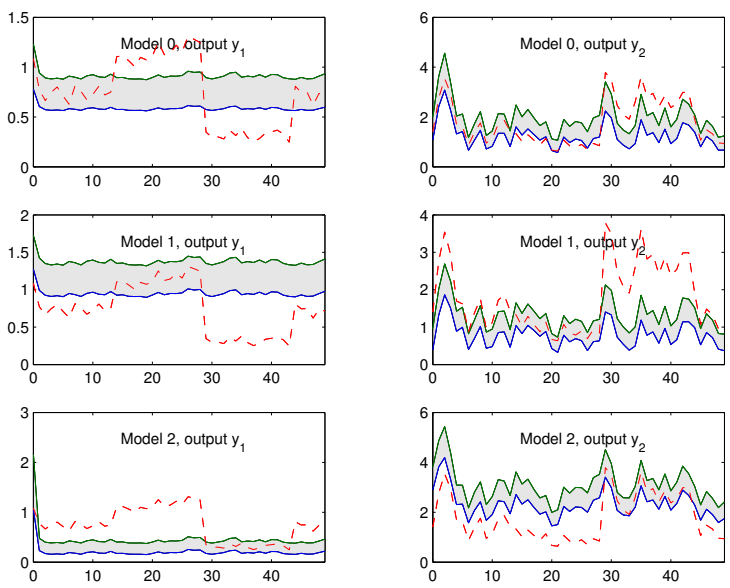

Fig. 3. Interval outputs estimated by the three models.

computed with the $i^{\text {th }}$ model:

$$
\begin{aligned}
& y_{i j}^{-}(k)=\inf _{y_{i j}(k) \in \mathcal{D}_{y, k, i}} y_{i j}(k) \\
& y_{i j}^{+}(k)=\sup _{y_{i j}(k) \in \mathcal{D}_{y, k, i}} y_{i j}(k)
\end{aligned}
$$

where $y_{i j}$ has been defined in (15). The figure 3 shows the bounds of the two outputs $y_{i 1}, y_{i 2}$ (in columns) of each model (in rows) computed thanks to (19). The simulation was done on the horizon $\left[\begin{array}{ll}0 & 50\end{array}\right]$, the changes of operating mode occured at the moments 15 (switching from $M_{0}$ to $M_{1}$ ), 40 (switching from $M_{1}$ to $M_{2}$ ) and 45 (return to the mode $\left.M_{0}\right)$. On each part of the figure, the output $y_{i m}(k)$ is drawn in dash-line in order to be compared with its estimates based on each model. Therefore the admissible output domains are defined for the three models. The active mode is determined by analyzing the measured output of the system together with these three domains. In the considered example, the output is corrupted by a bounded noise and can be directly compared with the bounds of the output interval of the three models. For each component $y_{m j}$ of the measured output and each model $M_{i}$, one defines the residuals:

$$
r_{i j}(k)=\left[y_{i j}^{-}(k)-y_{m j}(k), y_{i j}^{+}(k)-y_{m j}(k)\right]
$$

with $i=0,1,2, j=1,2$ and $y_{i j}^{-}$et $y_{i j}^{+}$being the lower and upper bounds of the output of the model $M_{i}$ (19). The residuals $r_{i j}$ are shown on figure 4. Clearly, it is possible to detect when each model presents an interval residual 

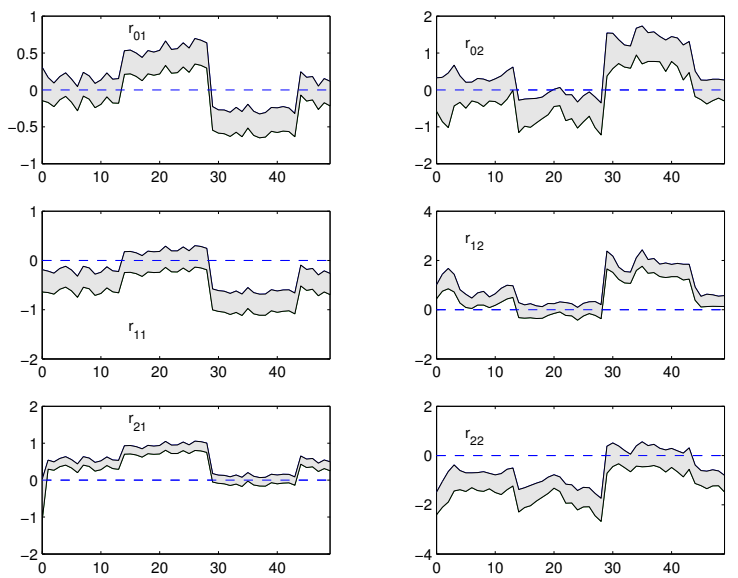

Fig. 4. Residuals issued from the three models.
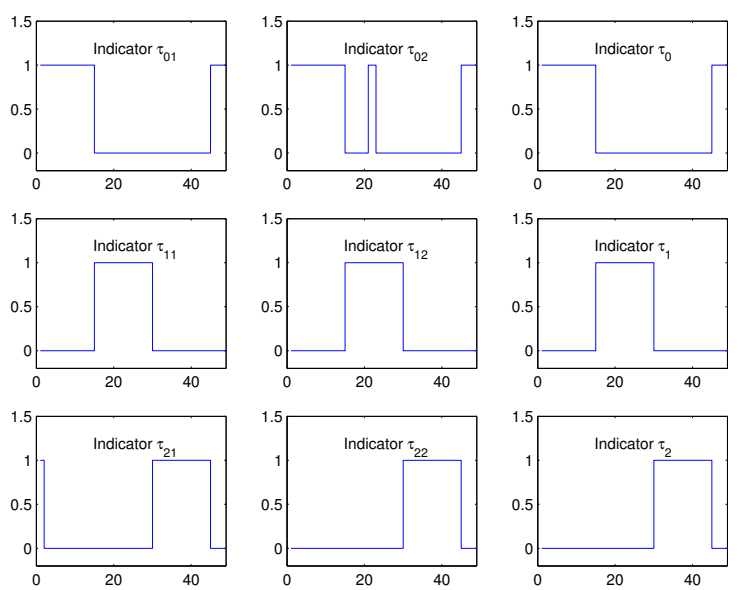

Fig. 5. Indicators of operating mode change.

containing the value 0 . Thus, the analysis of the residuals is based on the sign of the residual bounds:

$$
\tau_{i j}(k)=\frac{\left(1-\operatorname{sign}\left(y_{i j}^{-}(k)-y_{j}(k)\right)\left(y_{i j}^{+}(k)-y_{j}(k)\right)\right)}{2}
$$

where $i$ and $j$ respectively relates to the $i^{\text {th }}$ model and the $j^{\text {th }}$ component of the output. The $i^{t h}$ mode is declared active if all the components of the corresponding outputs are consistent with the $i^{t h}$ model, that is to say: if all the residuals $r_{i j}$ contain the zero value. Therefore, the residuals $\tau_{i}(k)$ are computed by the logic multiplication of the residuals $\tau_{i j}(k): \tau_{i}(k)=\tau_{i 1}(k)$ and $\ldots$ and $\tau_{i m}(k)$.

The residual evolutions are represented on the figure 5 , where $\tau=1$ (resp. $\tau=0$ ) testifies to (resp. invalidates) the membership of the origin to the interval residual. The obtained results are discriminating with respect to the changes of mode. The examination of $\tau_{01}$ and $\tau_{02}$ corresponding to mode 0 , enables to conclude that the mode 0 is active in the time intervals $\left[\begin{array}{ll}0 & 12\end{array}\right]$ and $\left[\begin{array}{ll}45 & 50\end{array}\right]$. The analysis of the residuals resulting from the two other models confirms and supplements this conclusion. The six graphs are coherent and enable to define the active mode, at every moment.

\section{CONCLUSION}

Undoubtedly, taking benefits of any knowledge about uncertainties is one of the fundamental points of current research and development in system analysis. This communication was focused on the bounded approach which uses a representation of each uncertainty by an interval. The propagation of the intervals along the time in the system equation results in defining interval observers, which provide interval estimates of the system state. Within the framework of the diagnosis, that leads to define fault indicators of the interval type. As a further research, the separability of the mode of functioning will be analyzed including the effects of noise, disturbances and outliers.

\section{REFERENCES}

[1] Alhaj-Dibo, M., Maquin, D. and Ragot, J. (2004) 'Data validation of uncertain dynamic systems', 15th International Workshop on Principles of Diagnosis.

[2] Armengol, J., Vehi, J., Travé-Massuyes, L. and Sainz, M.A. (2000) 'Interval model-based fault detection using multiple sliding time windows', 4th Symposium on Fault Detection Supervision and Safety for Technical Processes, Safeprocess'2000.

[3] Combastel, C. (2005). 'A state bounding observer for uncertain nonlinear continuous-time systems based on Zonotopes', 44th IEEE Conference on Decision and Control, pp.7228-7234.

[4] Frank, P.M. (1987) 'Diagnosis in dynamical Systems via state estimation - a survey', In: System Fault Diagnostics, Reliability and Related Knowledge- Based Approaches (Tzafestas, S. et al., Eds.), Vol. 1, pp.35-98, Reidel Publishing Company.

[5] Hou, M. and Muller, P.C. (1992) 'Design of observers for linear systems with unknown inputs', IEEE Trans. Autom. Cont., Vol. 37(6), pp.871-875.

[6] Jaulin, L., Kieffer, M., Didrit, O. and Walter, E. (2001), Applied Interval Analysis, Springer-Verlag, London.

[7] Kieffer, M., Jaulin, L. and Walter, E. (2002), Guaranteed recursive nonlinear state bounding using interval analysis, International Journal of Adaptive Control and Signal Processing, Vol. 16(3), pp. 193-218.

[8] Luenberger, D.G. (1965), 'Observers for multivariable systems', IEEE Trans. Autom. Cont., Vol. 11(2), pp. 190-197.

[9] Lunze, J., Steffen, T. and Riedel, U. (2003) 'Fault diagnosis of dynamical systems based on state-set observers', 14th International Workshop on Principles of Diagnosis, DX'03.

[10] Ploix, S., Adrot, O. and Ragot, J. (1999) 'Parameter uncertainty computation in static linear models', 38th Conference on Decision and Control.

[11] Puig, V., Quevedo, J., Figueras, J., Riera, S., Cembrano, G., Salamero, M. and Wilhelmi, G. (2003) 'Fault detection and isolation of rain gauges and limnimeters of Barcelona's sewer system using interval models', 5th Symposium on Fault Detection Supervision and Safety for Technical Processes, Safeprocess'2003, pp.621-626.

[12] Ragot, J., Maquin, D. and Adrot, O. (2006) 'Parameter uncertainties characterisation for linear models', 6th Symposium on Fault Detection Supervision and Safety for Technical Processes, Safeprocess'2006.

[13] Raïssi, T., N. Ramdani, Candau, Y. (2004) 'Set membership state and parameter estimation for systems described by nonlinear differential equations,' Automatica, 40(10), pp. 1771-1777.

[14] Slotine, J.J., Hedrick, J.K. and Misawan E.A (1987) 'On sliding observers for nonlinear systems', J. of Dynamic Syst., Measurement and Control, Vol. 109, pp.245-252.

[15] Stancu, A., Puig, V., Cugueró, P., Quevedo, J. (2005) 'Benchmarking on Approaches to Interval Observation Applied to Robust Fault Detection', In: Global Optimization and Constraint Satisfaction, pp. 171-191, Lecture Notes in Computer Science, Springer Verlag. 